\title{
Le cahier août-décembre 1927 de Catherine Pozzi
}

\section{Françoise Simonet-Tenant}

\section{OpenEdition}

\section{Journals}

Édition électronique

URL : http://journals.openedition.org/genesis/524

DOI : 10.4000/genesis.524

ISSN : 2268-1590

\section{Éditeur :}

Presses universitaires de Paris Sorbonne (PUPS), Société internationale de génétique artistique littéraire et scientifique (SIGALES)

\section{Édition imprimée}

Date de publication : 1 janvier 2011

Pagination : 155-176

ISBN : 978-2-84050-749-9

ISSN : $1167-5101$

Référence électronique

Françoise Simonet-Tenant, "Le cahier août-décembre 1927 de Catherine Pozzi », Genesis [En ligne], 32 | 2011, mis en ligne le 25 septembre 2012, consulté le 23 avril 2019. URL : http:// journals.openedition.org/genesis/524; DOI : 10.4000/genesis.524 


\title{
Le cahier août-décembre 1927 de Catherine Pozzi
}

\author{
Françoise Simonet-Tenant
}

$\mathrm{F}$

ille de Samuel Pozzi, fondateur de la gynécologie française, Catherine Pozzi est née en 1882. Élevée dans le giron de la haute bourgeoisie et de l'aristocratie parisiennes, Catherine Pozzi est l'auteur de quelques poèmes remarqués ${ }^{1}$, d'une nouvelle épistolaire et autobiographique, Agnès ${ }^{2}$ d'un traité resté inachevé, mi-poétique, mi-scientifique, Peau d'Âme $e^{3}$. Elle a tenu pendant son enfance et son adolescence, de 1893 à 1906 , un journal qu'elle a interrompu puis repris en 1913, après avoir pris conscience de l'échec de son mariage. Ce journal l'accompagnera jusqu'à la mort en 1934. Elle s'y montre une observatrice ironique et désabusée de la société mondaine dans laquelle elle évolue. Elle est aussi une tuberculeuse qui se débat avec la souffrance et qui vit perpétuellement avec l'idée d'un temps compté ; elle est une autodidacte insatiable également avide de philosophie et de chimie, de mathématiques et de biologie ; elle est enfin l'amante de Paul Valéry avec lequel elle noue, de 1920 à 1928, une liaison amoureuse et intellectuelle, passionnée et conflictuelle, aussi riche que dévastatrice. Dans son journal sont consignées les étapes de cette relation, et une abondante correspondance est échangée entre les deux amants. En janvier 1928, Catherine Pozzi rompt avec Paul Valéry pour s'enfermer dans le silence du journal dépossédé de son destinataire privilégié, prison de l'échec amoureux.

Le journal d'adulte tel qu'il a été édité par Claire Paulhan en 1987 chez Ramsay recouvre trente-six cahiers, trois carnets de petit format et dix liasses de feuilles non reliées. Les cahiers, carnets et liasses qui vont de janvier 1913 à avril 1929 ont été légués par Catherine Pozzi à la Bibliothèque nationale de France avec une clause de réserve de communication pendant les trente années suivant sa mort. Une partie de son journal était donc théoriquement accessible depuis décembre 1964. Le reste du journal a longtemps été conservé par la famille avant de faire l'objet d'un dépôt récent. En 2000 sont remises en dation à l'État les nombreuses archives que la famille de Catherine Pozzi possédait encore, comprenant, entre autres, des cahiers du journal, des cahiers de travail et un immense massif épistolaire.

L'édition critique du journal d'adulte établie par Claire Paulhan, texte de plus d'un million cinq cent mille signes, trouva suffisamment de lecteurs - alors que son auteur était encore quasiment une inconnue - pour épuiser un premier tirage de cinq mille exemplaires et pour nécessiter une seconde impression dès 1990 . Le texte est réédité en format poche en 2005 aux éditions Phébus avec révision de l'appareil critique. Quand Claire Paulhan s'est attelée à cette tâche d'édition, elle s'est trouvée confrontée à un texte dense, très long, parfois elliptique, parfois hermétique, parsemé d'allusions biographiques et historiques qu'il était nécessaire d'élucider pour le lecteur contemporain. Elle s'est alors fixé un objectif qu'elle résume vingt ans plus tard :

Paradoxalement, l'édition théoriquement idéale qui reproduirait de manière «diplomatique » ou « génétique » la forme du manuscrit originel s'avérait impraticable, dans ce cas comme pour les autres journaux intimes auxquels j'ai eu affaire depuis : trop difficile à lire, trop encombrée de signes typographiques inhabituels, qui parasitent la lecture. Or, il s'agissait bien de rendre lisible au plus grand nombre un texte jusque-là souterrain et donc, par principe, invisible. Chaque texte nécessite une appréhension particulière, une méthodologie originale

1. Seul, le poème Ave sera publié de son vivant (La Nouvelle Revue française, $\mathrm{n}^{\circ} 195,1^{\text {er }}$ décembre 1929, p. 757-758).

2. Agnès, La Nouvelle Revue française, $\mathrm{n}^{\circ} 161,1^{\mathrm{er}}$ février 1927, p. 155-179. 3. Peau d'Âme, Paris, Corrêa, 1935. Réimpression, avec préface et notes de Lawrence Joseph : La Différence, coll. «Philosophia Perennis », 1990. 
qui demande réflexion, qui demande également à être clairement décrite, par respect pour l'auteur, mais aussi pour le lecteur. Si j'ai rapidement saisi la difficulté intellectuelle et morale de ces publications d'autobiographies d'auteurs morts, je n'ai pas su rédiger, à l'époque, une « Note sur l'édition 4 » [...].

Ce repentir d'éditrice suggère, s'il est besoin de le rappeler, la transformation inéluctable subie par un journal manuscrit lors de son édition. Sans doute la stratégie éditoriale de Claire Paulhan était-elle alors pertinente : exhumant par son édition un texte invisible, elle le ressuscitait et le rendait accessible à un assez large public, séduit par un livre qui unit inextricablement le texte et la vie jusqu'à l'épuisement de l'un et de l'autre. La voie était également ouverte pour que chercheurs et universitaires, ces lecteurs de l'ombre, pussent aller voir de plus près comment s'articulaient les différentes pratiques graphiques et scripturaires dans les manuscrits. C'est ce que nous avons essayé de faire dans un précédent article de Genesis consacré au « journal comme laboratoire 5 »; c'est ce que nous tenterons de faire également ici, donnant à voir des pages du cahier illustré tenu à deux mains par Catherine Pozzi et Paul Valéry, absentes de l'édition publiée du journal de Catherine Pozzi. Nous remercions Catherine Bourdet et Martine Boivin-Champeaux, ayants droit de Catherine Pozzi et de Paul Valéry, d'avoir autorisé la reproduction de quelques pages de cet étonnant cahier6.

\section{Polymorphisme d'un espace d'écriture à deux plumes}

Lorsque l'on considère la liste des cahiers qui composent le journal de Catherine Pozzi, on constate l'existence de cahiers parallèles pour une même période : ainsi le cahier numéroté XX qui va de juillet 1927 au 12 janvier 1928 coexiste avec le cahier qui va d'août à Noël 1927. Ce dernier cahier, à la couverture cartonnée bleu-vert, $19 \times 29,5 \mathrm{~cm}$, comporte soixante-dix feuillets lignés, légèrement jaunis, et quatre pages de garde, sur papier rose buvard. Sur le verso de la page de couverture bleu-vert figurent les inscriptions suivantes à l'encre noire :
Du mois d'août 1927 à

Noël même année

Été 1927

Tous les dessins de ce cahier sont de Paul Valéry

CK

Karin

Entre les initiales CK et le prénom Karin, on devine quelques mots à l'encre noire qui ont été grattés et dont il reste des traces peu nettes, au déchiffrement conjectural : « à qui je donne ce cahier in memoriam ». $\mathrm{C}$ et $\mathrm{K}$ sont les initiales de Catherine et Karin - surnom donné par Paul Valéry et que Catherine Pozzi adopta à plusieurs reprises comme pseudonyme. Sur la couverture bleu-vert s'inscrivent d'ailleurs également à l'encre les initiales CK sous lesquelles sont dessinés un polygone et une présentation en miroir des lettres du prénom Karin :

$\begin{array}{cccc}\mathrm{K} & \mathrm{N} & & \mathrm{K} \\ & & \text { A I A } & \\ & & \mathrm{R} & \\ \mathrm{N} & \mathrm{K} & \text { I A I } & \\ & & \mathrm{N}\end{array}$

Les soixante-dix feuillets que comporte le cahier sont tous occupés : quarante-six pages présentent des aquarelles de diverses couleurs ; trente et une pages, des dessins ou griffonnages faits à la mine de plomb, à la plume et au lavis ; soixante-deux pages sont entièrement dévolues au texte ; sur une page $\left(\mathrm{f}^{\circ} 25 \mathrm{v}^{\circ}\right)$ est collée la photographie d'un bouddha. Dans le cahier sont glissées (non collées) treize pièces diverses : deux cartes postales, une carte de visite, un article de presse et des

4. Mireille Diaz-Florian, Catherine Pozzi. La vocation à la nuit, Paris, éditions Aden, 2008, p. 11-12.

5. Françoise Simonet-Tenant, « Catherine Pozzi, le Journal comme laboratoire de l'œuvre », Genesis, n 16, «Autobiographies », 2001, p. 75-96. 6. Ce cahier figure donc dans le fonds Pozzi de la BnF, fonds encore en cours de classement. 
feuillets divers et hétéroclites (des notes sur «Platondualisme », des notes scientifiques, une copie tapuscrite du poème «Vale », un feuillet où figurent des plans de table, une carte où sont copiés des titres d'ouvrages scientifiques, un feuillet à l'en-tête de «l'Université de Paris/Faculté des Sciences/Évolution des êtres organisés 105 Boulevard Raspail VI » où figurent de la main de Catherine Pozzi deux dessins de préparation et l'appréciation chiffrée du correcteur : «De sérieuses qualités d'observateur. Faites un effort pour dessiner un plus grand nombre de préparations $-12 » .$. ). Cinquante dates sont présentes dans le cahier du 19 août 1927 au 25 décembre 1927. Ces dates peuvent être d'une grande précision : «26.8.27 after dinner » $\left(\mathrm{f}^{\circ} 10 \mathrm{r}^{\circ}\right)$, « 26 Nov. Morning » $\left(\mathrm{f}^{\circ} 54 \mathrm{r}^{\circ}\right)$, « 10 Déc. 3 h. » $\left(\mathrm{f}^{\circ} 62 \mathrm{r}^{\circ}\right) \ldots$ On a l'impression, à certaines pages, que les dates ont été apposées par Catherine Pozzi légèrement après coup, comme s'il s'agissait de certifier par la date l'authenticité d'un dessin réalisé de la main de Valéry (au folio $34 \mathrm{r}^{\circ}$ où est peinte une aquarelle : «Par P. V. le 29.10.27 à 6-7 heures du soir »), l'existence d'une conversation ou un propos dicté par Paul Valéry à Catherine Pozzi. Le temps s'invite sans cesse dans ce cahier, que ce soit la date des moments partagés, concrétisés par un dessin ou une idée jetée sur le papier, ou la mention prospective, à la manière de l'agenda, des occupations d'un des deux scripteurs (voir fig. 7, $\left.\mathrm{f}^{\circ} 16 \mathrm{r}^{\circ}\right)$.

Il est manifeste que ce cahier parallèle est plus particulièrement dévolu à la notation des conversations avec Paul Valéry, espace d'échange entre deux plumes. Il s'agit sans doute de ce que Catherine Pozzi appelle le « cahier à dessins » :

P. V. était ici comme tous les matins, hier, et la page du cahier à dessins porte témoignage d'une heure bien vivante. Mais l'extraordinaire, - non pas l'extraordinaire, l'étonnant qui est ordinaire -, c'est qu'à peine je suis, à peine je vais dans une région de pensée, que, lui, il y va de même et ceci sans être prévenu. Nous en sommes à un synchronisme tel que l'esprit de l'un donne le même son que l'esprit de l'autre, tous renseignements de langage inutiles : au point que nous ne pouvons pas, du point de vue de l'esprit, essayer d'être à part. Lui va plus loin que je ne vais, sauf d'un côté, mais c'est moi qui pars cependant la première. J'apporte la faim et prends l'élan?.
Si l'on ne peut parler ici de journal épistolaire, le cahier à dessins semble remplir le rôle d'un journal conversationnel aux divers visages. Rappelons leurs adresses respectives : Paul Valéry habitait rue de Villejust et Catherine Pozzi, rue de Longchamp, deux rues du $\mathrm{XVI}$ e arrondissement fort proches l'une de l'autre. Valéry venait fréquemment chez Catherine Pozzi, le matin, pour converser à bâtons rompus sur des sujets intellectuels variés (la politique, les mathématiques, le langage, la mémoire, le temps...) : peut-être y a-t-il là pour Valéry, avec une interlocutrice à sa mesure, un prolongement dialogique du travail de réflexion qu'il mène dès l'aube dans ses propres Cahiers. De cette venue de Paul Valéry au domicile pozzien témoigne l'aquarelle présente au folio $1 \mathrm{v}^{\circ} \mathrm{du}$ cahier, accompagnée d'un commentaire pour le moins acide de Catherine Pozzi (voir fig. 2). Plusieurs passages écrits de la main de Catherine Pozzi portent le commentaire marginal «Dicté » (voir fig. 5, $\mathrm{f}^{\circ} 5 \mathrm{r}^{\circ}$ ). D'autres portent la trace allusive et elliptique des conversations ou des échanges à bâtons rompus. Ainsi une double page est couverte de notes en tous sens dont trois sont précédées par les lettres a, b, c, et la diariste précise avec minutie : "Les 3 lettres au-dessus de ces bouts de conversation ne concernent pas leur liaison logique mais leur ordre dans le temps. $\mathrm{CK} \gg\left(\right.$ voir fig. 13 et $14, \mathrm{f}^{\circ} 66 \mathrm{v}^{\circ}$ et $67 \mathrm{r}^{\circ}$ ); quelques pages sont consacrées à la copie de notes puisées dans les « Cahiers de V 1922 » (du folio 17 au folio 20), ce qui témoigne d'une étonnante circulation entre les cahiers valéryens et les cahiers pozziens. Ces pages donnent une idée des sujets privilégiés par les deux interlocuteurs : les conversations sont avant tout scientifiques ou philosophiques, bien plus que littéraires. Néanmoins l'esprit de sérieux ne règne pas tout-puissant sur ce cahier : les écritures et dessins ludiques et grinçants ne sont pas en reste. Le cahier s'ouvre sur un texte mi-hermétique, mi-sarcastique comme les affectionnait Catherine Pozzi, suivi d'une citation de vers de Robert Browning (voir fig. 1) ; les vers de mirliton apparaissent, ici et là, ainsi que des dessins ironiques (voir fig. 8, $\mathrm{f}^{\circ} 39 \mathrm{r}^{\circ}$ ).

7. Catherine Pozzi, Journal 1913-1934, Paris, Phébus, coll. « Libretto », 2005, p. 425-426 (7 décembre 1927). 
Ce cahier n'est pas le seul à abriter des dessins de Valéry qui manifeste ainsi sa présence dans l'intimité pozzienne. On observe en particulier, dans plusieurs cahiers de 1926 et 1927, un assez grand nombre de dessins et, parmi eux, plusieurs représentations de Catherine Pozzi (au piano, allongée sur un lit, en train de lire...). On connaît par ailleurs l'importance que joue le dessin dans les cahiers de Valéry8 et sa propension à s'autoportraiturer et à dessiner ses mains (la droite en train d'écrire, la gauche tenant une cigarette). Valéry acclimate donc dans le journal pozzien la pratique qui est la sienne dans ses propres Cahiers.

\section{Micro-lecture d'une page $\left(f^{\circ} 55 r^{\circ}\right)$}

Sur cette page (voir fig. 11) figurent une réflexion programmatique sur le langage ( La 1 re quest. serait de redéfinir ce que les grammairiens appellent les parties du discours »), des vers de mirliton dédiés à la princesse de Bassiano, un schéma, un dessin légendé (encre et aquarelle) qui représente une main et des notes proches de celles d'un agenda.

En face de la réflexion sur le langage est inscrite la mention : «V Langage ». L'initiale renvoie à Valéry et l'on peut imaginer qu'il s'agit là d'un propos de Valéry, noté par Catherine Pozzi. La diariste et le poète s'exercent constamment à la définition des termes employés. Refondant perpétuellement le dictionnaire qu'ils composent à leur usage, ils sont toujours à la recherche du terme le plus exact, de la formulation la plus dense et la plus précise possible : «Vous trouvez une question à demander aux choses, et je la finis, et nous la répétons en plus juste formule9. " Réunis par un même goût du dialogue et de la définition où l'on savoure la volupté de l'intelligence, ils sont passionnés par la question du «Langage ». Valéry en fera une des rubriques de ses Cahiers, lors du second classement de ses notes à partir de 1921, quand il est repris par le désir d'y mettre de l'ordre et d'en dégager les idées essentielles, et l'on sait que Catherine Pozzi a participé à la mise en œuvre de ce second classement. L'édition réalisée en 1973 par Judith Robinson pour la collection de la Pléiade des Cahiers de Valéry donne une idée de ce qu'aurait pu être un ordonnancement thématique des fragments et notes de Valéry. La section «Langage » livre maintes réflexions qui mettent en évidence les relations privilégiées entre main et langage 10 , une main que Valéry dessine sur la page même où il réfléchit sur le langage.

Les échanges intellectuels intenses entre Paul Valéry et Catherine Pozzi laissent parfois la place, comme autant de respirations nécessaires, à des plaisanteries et pochades où l'imagination débridée se laisse aller à toutes les fantaisies. C'est sans doute l'un des rôles remplis par les vers consacrés par Catherine Pozzi à l'« infusoire », protozoaire nageur à cils vibratiles. Autodidacte acharnée, elle a obtenu, en octobre 1927, la deuxième partie du baccalauréat et, forte de ce succès, elle suit des cours à la Sorbonne ainsi qu'à l'Institut Pasteur et au Collège de France. Son journal mentionne souvent à cette époque ses réactions après qu'elle a assisté aux cours de biologie de Picard et Caullery. Dans ce contexte, les vers sur "l'infusoire» semblent moins étonnants! Que ces vers soient dédiés à Marguerite de Bassiano, dédicace fantaisiste et ironique, méritent quelque explication. En février 1927, Catherine Pozzi a publié, dans la revue de La NRF, Agnès, une nouvelle autobiographique et épistolaire. La nouvelle, dont Catherine Pozzi voulait la publication anonyme, avait d'abord été proposée par Paul

8. Sans doute le dessin peut-il revêtir plusieurs significations : dessins de circonstances où sont brossés les lieux de l'écriture, dessins où est mis en scène l'acte d'écriture, dessins qui participent au processus génétique, constituant une étape dans le développement de l'idée et accompagnant les flux et reflux de la pensée, dessins qui suggèrent ce qui ne saurait se figer dans les mots. Voir S. Bourjea, «Écriture et Dessin dans les premiers "Cahiers" de Valéry », Forschungen zu Paul Valéry/Recherches valéryennes, $\mathrm{n}^{\circ}$ 14, Autobiographie und Autoportrait bei Valéry, 2002, p. 100 et p. 114 : « la coprésence du langage verbal et du dessin, sur la page des écritures du cahier, est bien entendu fondamentale... » « Il faudrait en fait étudier, au sein même de l'élaboration des cahiers, toute une stratégie de dérives, à la fois de démultiplication, des langages utilisés et, corollairement, leur contamination, leur "déconstruction" réciproques, les écritures qui en naissent qualifiant la poïesis selon Valéry, si Poésie est pour lui, fondamentalement, ce qui échappe - ou doit échapper - à la rationalité discursive. »

9. Catherine Pozzi, Journal 1913-1934, op. cit., p. 192 (12 mai 1921).

10. Voir P. Valéry, Cahiers I, Paris, Gallimard, coll. « Bibliothèque de la Pléiade », 1973, p. 427 : «La main et le langage articulé sont profondément liés. L'animal manque des deux ensemble. Le geste - la communication par la vue. » 
Valéry à la princesse de Bassiano, commanditaire de la revue Commerce (1924-1932), cahiers trimestriels dirigés par Paul Valéry, Léon-Paul Fargue et Valery Larbaud. Marguerite de Bassiano, peu désireuse de publier une nouvelle anonyme dans sa revue et peu séduite par le texte, l'avait cédée à Jean Paulhan, prêt à la publier dans La NRF. Si ce lieu de publication convenait à Catherine Pozzi, elle n'en gardait pas moins un certain ressentiment vis-à-vis de la princesse, qui s'exprime par le biais d'une dédicace loufoque.

Sous les vers apparaît la main de Catherine Pozzi dessinée par Valéry. Le dessin est daté (« Morning 29.11.27 ») et légendé : « My hand by Testis ». Testis est l'un des surnoms que Catherine Pozzi donne à Valéry par allusion à La Soirée avec Monsieur Teste. Les surnoms imaginés par Catherine Pozzi pour Valéry sont très nombreux et accompagnent l'évolution de leur relation : des surnoms de l'amour (Lionardo et Tristan) et de ceux révélateurs de son admiration intellectuelle (Testis) à l'onomastique de la vengeance pratiquée par la diariste qui ne tolère ni les concessions du poète à la comédie sociale (« Bonheur-des-oies », « 30-ans-de-thés »), ni ce qu'elle juge être son absence de conscience morale (« le Monstre », « Hell », « Enfer », « le Diable »).

$\mathrm{Au}$ bas de la page figurent quelques notes qui ont tout de l'agenda :

Jeudi académie

Vendredi - conférence $3^{\mathrm{h}}$ à $5^{\mathrm{h}}$ prix Moréas

Samedi Sorbonne $10^{\mathrm{h}}$ matin déjeuner Bogart et Monod dîner France-Amérique
Selon toute vraisemblance, on a là l'emploi du temps de Paul Valéry, entré à l'Académie française en juin 1927. Le vendredi 2 décembre 1927, Valéry a effectivement donné une conférence aux Annales. Quant à «Bogart et Monod »avec lesquels Valéry déjeune le samedi, ce sont Julien-Pierre Monod, ami et admirateur de Valéry, qui constituera une importante collection de manuscrits, documents et éditions originales de Valéry (actuellement conservés à la Bibliothèque littéraire Jacques Doucet) et, très probablement, Ludo van Bogaert, neurologue belge, auquel Valéry fait allusion dans les Cahiers.

On observera seulement que la main dessinée sur cette page ne tient rien, et qu'il n'existe pas dans ce cahier de représentation de Catherine Pozzi écrivant, comme si s'inscrivait obliquement, par le biais du dessin, ce qui sera le motif d'une douleur bientôt insupportable pour la diariste : la non-reconnaissance par Valéry de son statut d'écrivain. «Je sens seulement, glacée, isolée, que j'ai probablement du talent, qu'il faut écrire, et que Valéry ne me l'a jamais dit ${ }^{11}$. » On ne manquera pas de se souvenir que cet étonnant cahier, qui fourmille d'échanges et de portraits de Catherine Pozzi, couvre les six derniers mois de leur liaison, dont la rupture sera effective en janvier 1928.

11. Catherine Pozzi, op. cit., p. 412 (5 août 1927). 
Laisean touth quoique fabuleux existe.
U. cagne du bec a le fenétre, mais tu cóté intirienr.

te bec puissant te ficke dous prose, vers, bouquins,

papiers, se redresse, ot chanke forrilles i' 'mivere.

les griffes aigïes cherchent Dam lo plumege poux ot

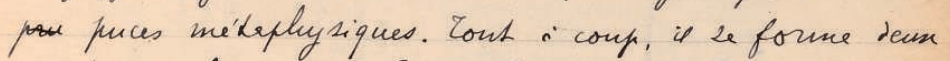

on hois abimes de Pascal dam l'atmospheie.

un abivine de Vascal" est comme un toud'aci en aviction.

mais moins dangereux. An fond de $P$ abine, Conth

songe qu'd ex l'heure du caf' an lait. Abime ct cafe'

un lait te refprochent, fomment in certain sysléme

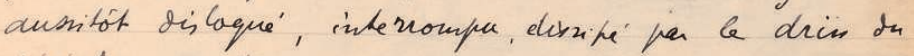

lelephone. 't'oisean reven an zeio, reconnunce

et une furie de tavail, une arbeur: coustuine 20 nid

de notions zperciales beforide...

Fig. $1: \mathrm{f}^{\circ} 1 \mathrm{r}^{\circ}$

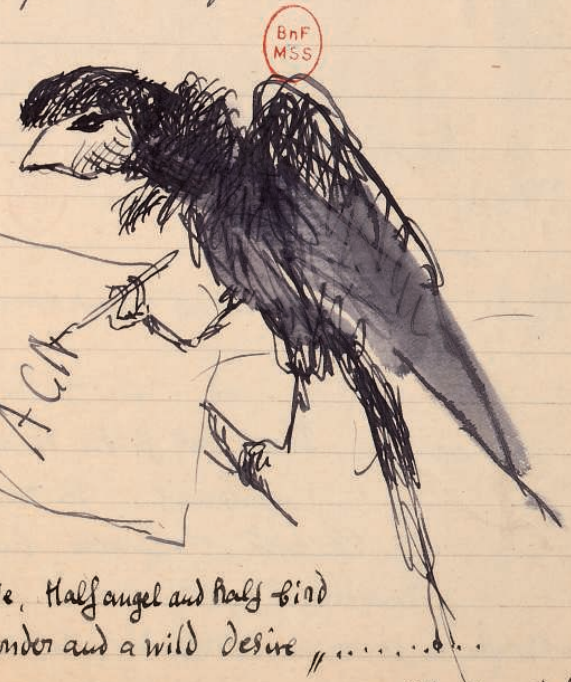

or: diffent pots har diffent means.

Confer. "O \& grie Love, Half angel and hald bind

and aft a wonder and a wild desive f.........

Novum Testamentum T $\theta$

L'oiseau Touth quoique fabuleux existe. Il cogne du bec à la fenêtre, mais du côté intérieur. Le bec puissant se fiche dans prose, vers, bouquins, papiers, se redresse, et chante pouilles à l'univers. Les griffes aiguës cherchent dans le plumage poux et prut puces métaphysiques. Tout à coup, il se forme deux ou trois abîmes de Pascal dans l'atmosphère. Un « abîme de Pascal » est comme un trou d'air en aviation, mais moins dangereux. Au fond de l'abîme, Touth songe qu'il est l'heure du café au lait. Abîme et café au lait se rapprochent, forment un certain système aussitôt disloqué, interrompu, dissipé par le drin du téléphone. L'oiseau revenu au zéro, recommence et une furie de travail, une ardeur à construire son nid de notions spéciales le possède...

Confer. « o Lyric Love, Half angel and half bird / and all a wonder and a wild desire ${ }^{1} » . . . .$.

or : different poets have different means ; as you like it.

1. Poème de Robert Browning. 


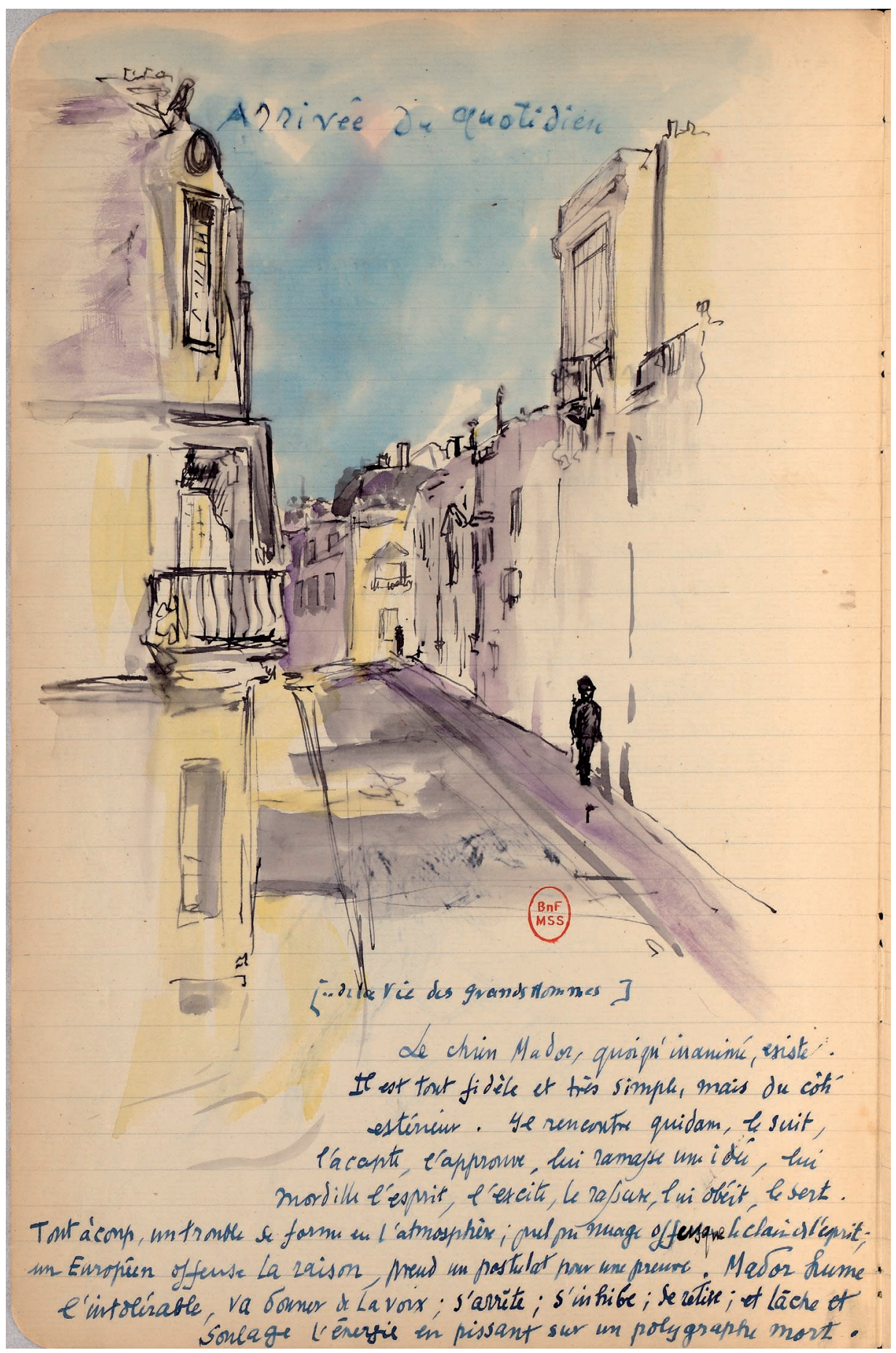

Fig. $2: f^{\circ} 1 v^{\circ}$

Arrivée du quotidien²

[...de la vie des grands hommes]

Le chien Mador, quoiqu'inanimé, existe. Il est tout fidèle et très simple, mais du côté extérieur. Il rencontre quidam, le suit, l'accepte, l'approuve, lui ramasse une idée, lui mordille l'esprit, l'excite, le rassure, lui obéit, le sert. Tout à coup, un trouble se forme en l'atmosphère ; quelque nuage offusque le clair et l'esprit ; un Européen offense la raison, prend un postulat pour une preuve. Mador hume l'intolérable, va donner de la voix ; s'arrête ; s'inhibe ; se retire ; et lâche et soulage l'énergie en pissant sur un polygraphe mort.

2. Un des surnoms donnés par Catherine Pozzi à Paul Valéry. 
19.8.27.

$$
\begin{gathered}
\text { Jugement - } \\
\text { dicté } \\
\text { égale } \neq \text { interjection }
\end{gathered}
$$

Je constate qu'il y a « quelque chose » initiale qui peut amener une expression.

\section{La question est la suiv :}

Il y a des cas où cette express reste ce qu'elle est

D'autres où elle fait partie d'une suite de transformations dont les unes sont des images, les autres, d'autres expressions or il arrive ds. certains cas que cette suite

remarquer à quel point PV parle naturellement facil $^{\mathrm{t}}$, le langage abstrait.
ce qui caractérise
à ce sujet je dirai que : $\alpha \quad$ Le mot « expression » c'est son rôle purement transitif. [au sens de la théorie des groupes]
$\beta \quad$ La soi disant analyse par les philosophes de ces suites de substitutions, les a conduits à hypostasier [c'est à dire à faire un sort....] aux expressions et à la forme des ex. sous le nom de « Jugement». Il suffit de voir de transformat. aboutisse à une express. telle que l'état initial [ou l'un des ét interméd. pris comme origine] soit en relation ${ }^{\circledR}$ avec la signification de l'expression finale. Si cette chaîne d'express satisfait à cert. condit. de conservation on aura ce que l'on appelle un raisonnement dont la dernière express sera dite la conclusion Nota très important J'ai dit « la dernière expression » ou conclusion ; ceci n'a de sens précis que ds le cas où le sujet prévoyait d'avance l'existence d'un terme dernier.$$
\text { « Jugement } \gg \text { Ill suffit de voir }
$$

(1) Sur ce seul passage « relation » il faudrait des pages 
C A THERIN E P OZZI-PA U L VALÉ R , C A H I E R 1927

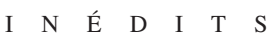

iq. $8 \cdot 2 z$.

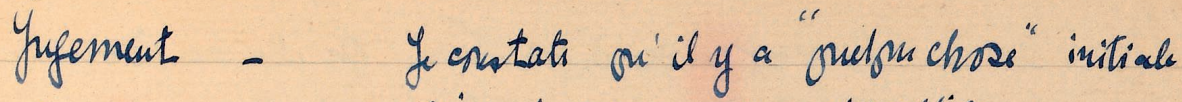

Sictí oni pent amener une exprepsion.

La preotion at la Juir:

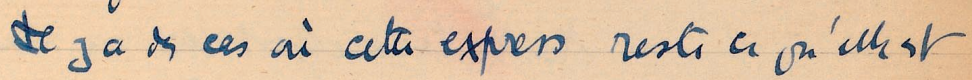

éalef interjection 1

Q 'autrs oi elu fail partis J'use suif a trausformations dout is unes sout of ineges, las auty, d'autres exprension

remargner a or i'arrive ds. errtains cas pu cets snits oul point de trausormat. abontine i un expen. Tells gu. Pr parle l'état initial [on l'un ds it intermid. pris conm natureblement orifin I si't as selation are la signification de tacit, le t'expossion finals. Si ceth chain D'express laufage satisfait icert. convit. A consenation on aura abstrait. in om c'on appeth un raisonnemut four la demion etpres sear vit la carclusics Nota Fi importañ y aidit "la dermién expersion" ri conchusin; Ceci $x^{\prime}$ a do sus pricis onits.h cas ori te ujer 'Mivojoit javance

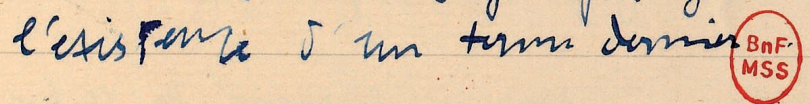

a pi caractérise

àasujet njovaigu:d te mot "expression" c'est son róle purement transitif. [au seus or la the stie os sroups]

B La soidisont analyse nar les philosophes de ces suites of substitutions, les a conduits a hypostasier - c'est à dise à fair un sort...j aix expretsions et à la farm des ex. Sous h nom de "Yusement". se suffir de vois

(4) Sur a seul passag" relation "it fanirait des pages

Fig. $3: \mathrm{f}^{\circ} 4 \mathrm{r}^{\circ}$

163 
le vague des définitions qu'ils en donnent

pr. s'assurer qu'il s'agit d'un cas de

mythol - car, et c'est très remarqu.,

tout leur effort est en sens contr. de ce qu'<i $>$ il est raisonnable et probable en fait de pysiologie du langage. C'est pourquoi les voit on inventer tout ce qu'il leur faut pour que leur fameux Jugement existe ; en part. les concepts, et les concepts mêmes les obligent à inventer les catégories.

«Langage des philosophes est technique mais n'est pas défini »

....... « ouïlle ! » est un jugement. « M... » est un jugement. Dès qu'une sensation donne naissance à un fait $\psi y c h$. de consci ${ }^{\text {ence }}$, il y a un jugem ${ }^{\mathrm{t}}$ qui se produit.

Ce qui a touj. embarrassé la pilos, ce sont les jugements universels - comment l'on pourrait s'élever au général - Mais c'est que le général n’est pas le général.

on s'élève de l'idée d'un triangle dessiné à l'idée de tr. - Il est remarqu. que tout de notre usage est fondé sur une image partic. de triangle. Or notre prétendue généralité consiste dans notre pouvoir [ou illusion de pouvoir] négliger, 
C A The RIN E P OZZI-PA U L VA LÉ R Y, C A H I E R 1927

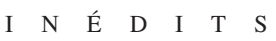

le ragne des difinition on "ils en dounent pr. S'assuver on'il s'afit J'un cas de mythoe - car, et c'ast tris remargu., tout leur eftort est en sens conts. of a guilth ast naidounaor it probabe en fait de Gysiologie on laufar. C'at pouronsi les riton inventer tout a on'il leur faut pour om len famur Yugenant etist ; en part. les concepts, et les coneepts meñs les soligents à unventer les eatigovies.

"Langafe des philosontes

est tichnipre mais $x^{\prime s}$

pes difini"
... "oviffe! "est un jugemint. M..."est mijufemut. Dis g'un Seusation Donne naissawa à un fait Yych. or consci." it $y$ a un jugemt on se prodvit Bnf-

A pri a tonj. eubarrara' la Gilos, a jout les juferments universels - communt I'n powail s'ilerer an finéral - Mrai c'at om le ginimal $n$ 'est pas a ginéral.

ons'élèr or l'idé d'un triagh destim' à l'ida'ch tr. - Se as remarph. ou toutenotre esagr est tous' our un iman partic. de triaugh. or notr prifeudu ginélatit' consist dae noth pourdis [on illusion ar pouvis $]$ nifliger,

Fig. $4: \mathrm{f}^{\circ} 4 \mathrm{v}^{\circ}$

165 


\section{Dick'}

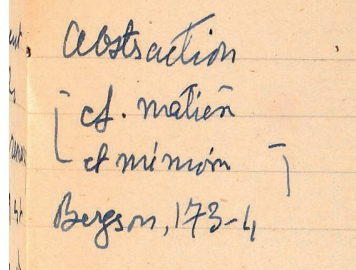

Tenir ps. mulh, um partí des proprifites or des pralitis or l'objet. Some l'abstraction se rattach essentiel I à a porevoin oir est disivis ds

a pi 'is y a de ples concret, et pirs l'act.

or l'ach a ces deut propriites de s'opéres

un moyen óofjets plus complexes one hic, ipar ex. jeter une pierre en nifligeant sa conleur - it de soperer arec un grauliti' o'objts diff'r griout une onalit' commum.

\section{L'ach est un traves formation a' unc Seuh variabh}

Fig. $5: f^{\circ} 5 r^{\circ}$

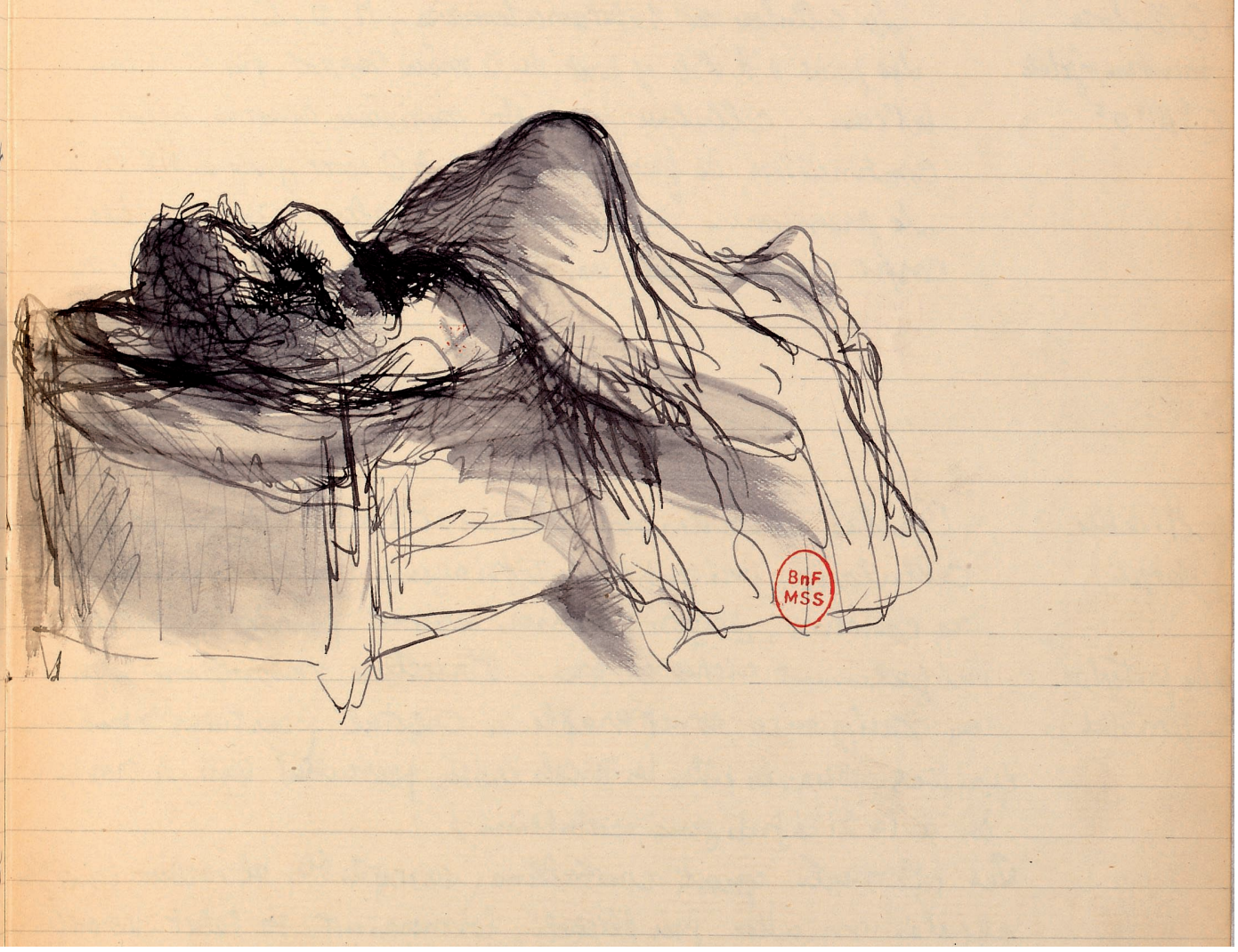

Dicté

[marge gauche] Abstraction [cf. matière et mémoire Bergson, 173-4] / [corps de texte] tenir pr. nulle, une partie des propriétés ou des qualités de l'objet. Donc l'abstraction se rattache essentielt à ce pouvoir qui est dérivé de ce qu'il y a de plus concret, et qui est l'acte. Or l'acte a ces deux propriétés de s'opérer au moyen d'objets plus complexes que lui, [par ex. jeter une pierre en négligeant sa couleur.] et de s'opérer avec une quantité d'objets différ. qui ont une qualité commune. L'acte est une transformation à une seule variable. 


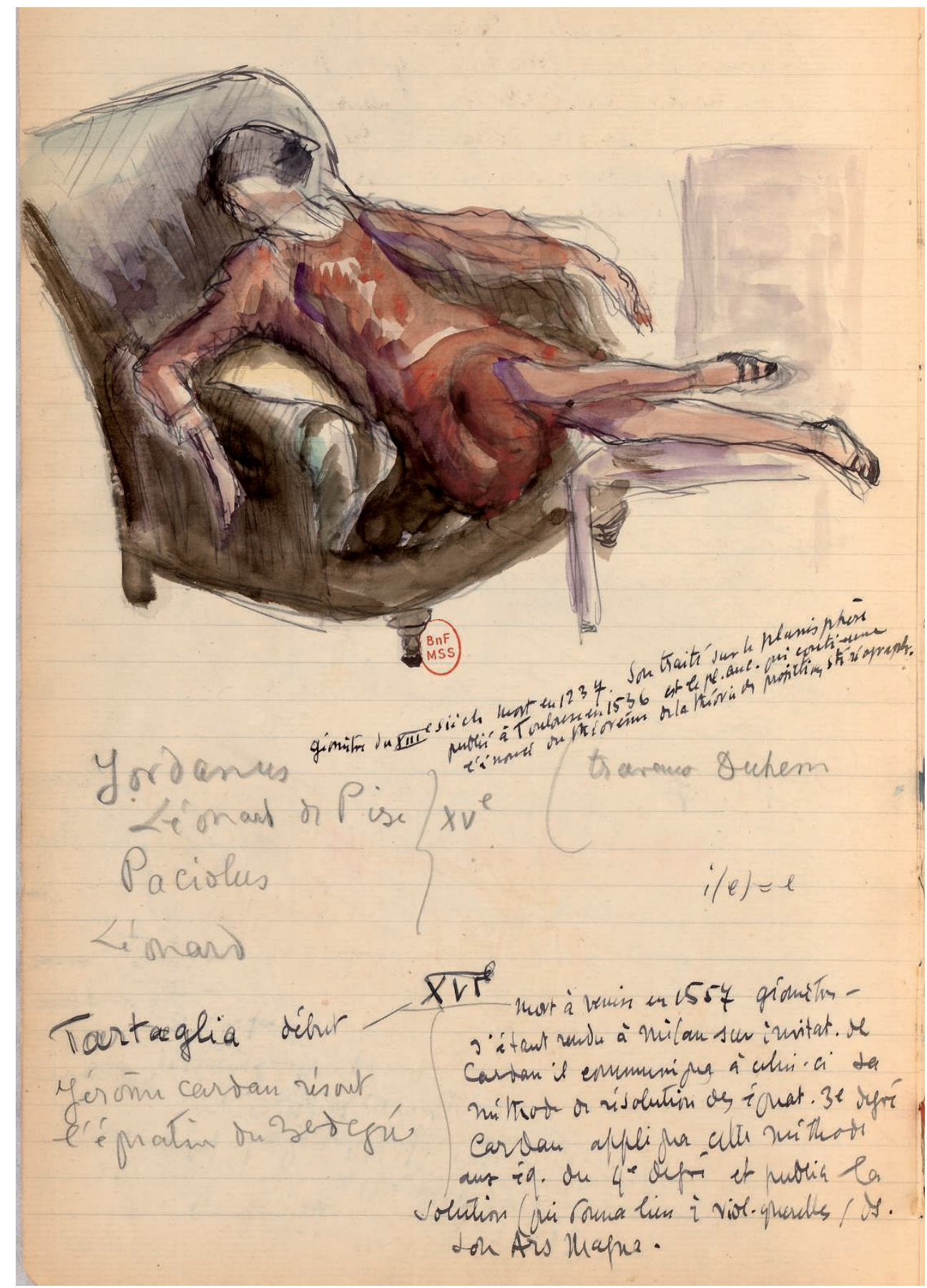

Géomètre du XIIIe siècle mort en 1237. Son traité sur le planisphère publié à Toulouse en 1536 est le pl. anc. qui contienne l'énoncé du théorème de la théorie des projections stéréograph.

(travaux Duhem

Jordanus
Léonard de Pise
Paciolus
Léonard
Tartaglia début
Jérôme Cardan résout
l'équation du 3e degré

$$
\mathrm{i}(\mathrm{e})=\mathrm{e}
$$

mort à Venise en 1557 en 1557 géomètre - s'étant rendu à Milan sur invitat. de Cardan il communiqua à celui-ci sa méthode de résolution des équat. 3e degré Cardan appliqua cette méthode aux éq. du 4e degré et publia la solution (qui donna lieu à viol. querelles) ds son Ars Magna. 


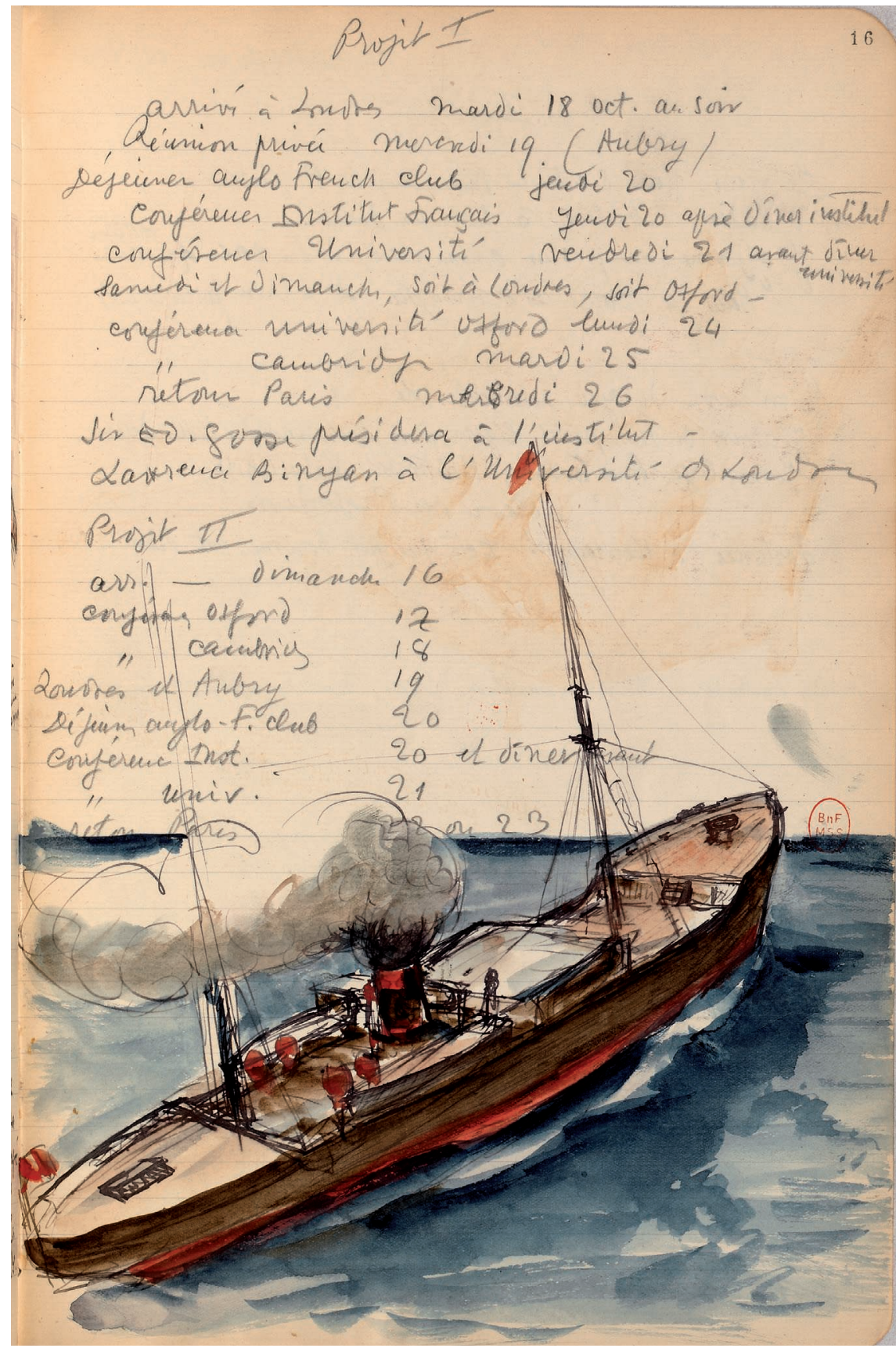

Fig. 7 : $\mathrm{f}^{\circ} 16 \mathrm{r}^{\circ}$

Projet II

arr. - dimanche 16

conférences Oxford 17

“ Cambridge 18

Londres et Aubry 19

Déjeuner anglo-F. club 20

Conférence Inst. $\quad 20$ et dîner [ill.]
Conférence Université Oxford lundi 24

" Cambridge mardi 25

retour Paris mercredi 26

Sir Ed. Gosse présidera à l'institut.

Lawrence Binyan à l'Université de London. 


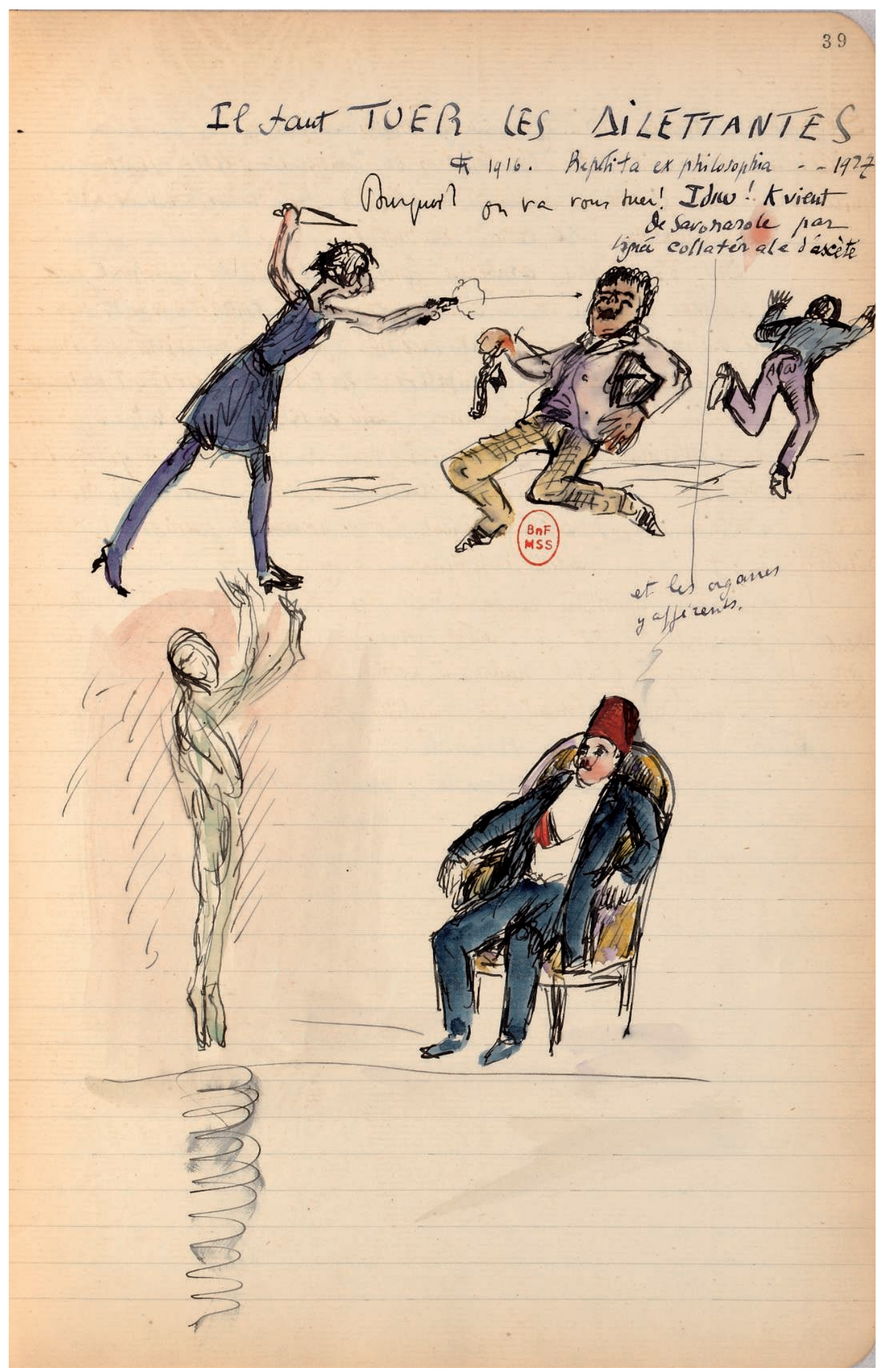

CK 19i6. Repetita et philosophia.. 1927

Il faut TUER Les DiletTANTES

Pourquoi ? on va vous tuer ! I $\delta \omega \omega$ ! K vient de Savonarole par lignée collatérale d'ascète $\rightarrow$ et les organes y afférents 


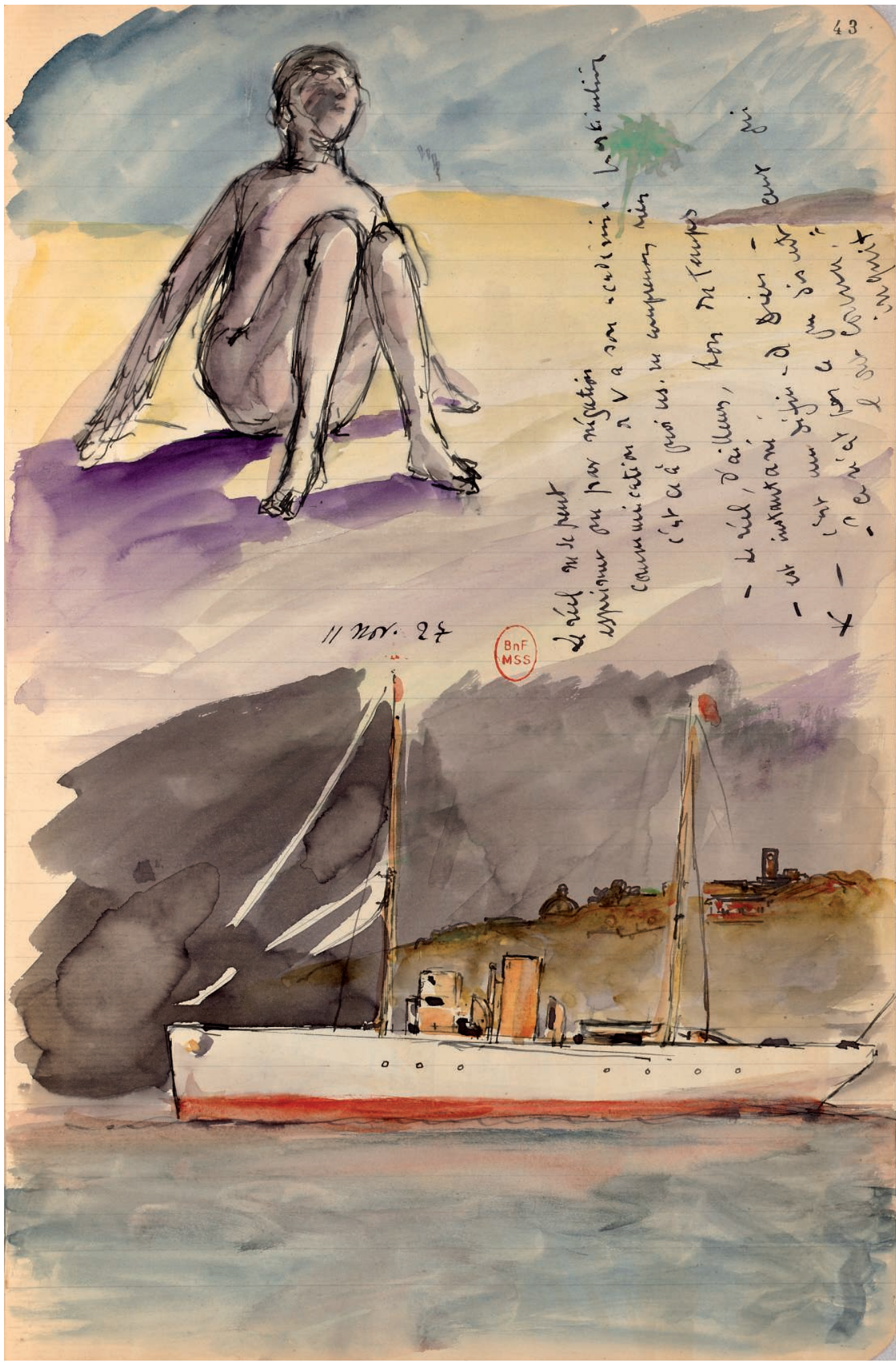

Fig. 9 : $f^{\circ} 43 r^{\circ}$

11 nov. 27

Le réel ne se peut / exprimer que par négation / Communication de V. à son académie particulière / C'est ce à quoi ns ne comprenons rien / - Le réel, d'ailleurs, hors du temps / - est instantané / K - c'est une défin. de Dieu - / - « Ce n'est pas ce que disaient ceux qui l'ont connu » / inquit 


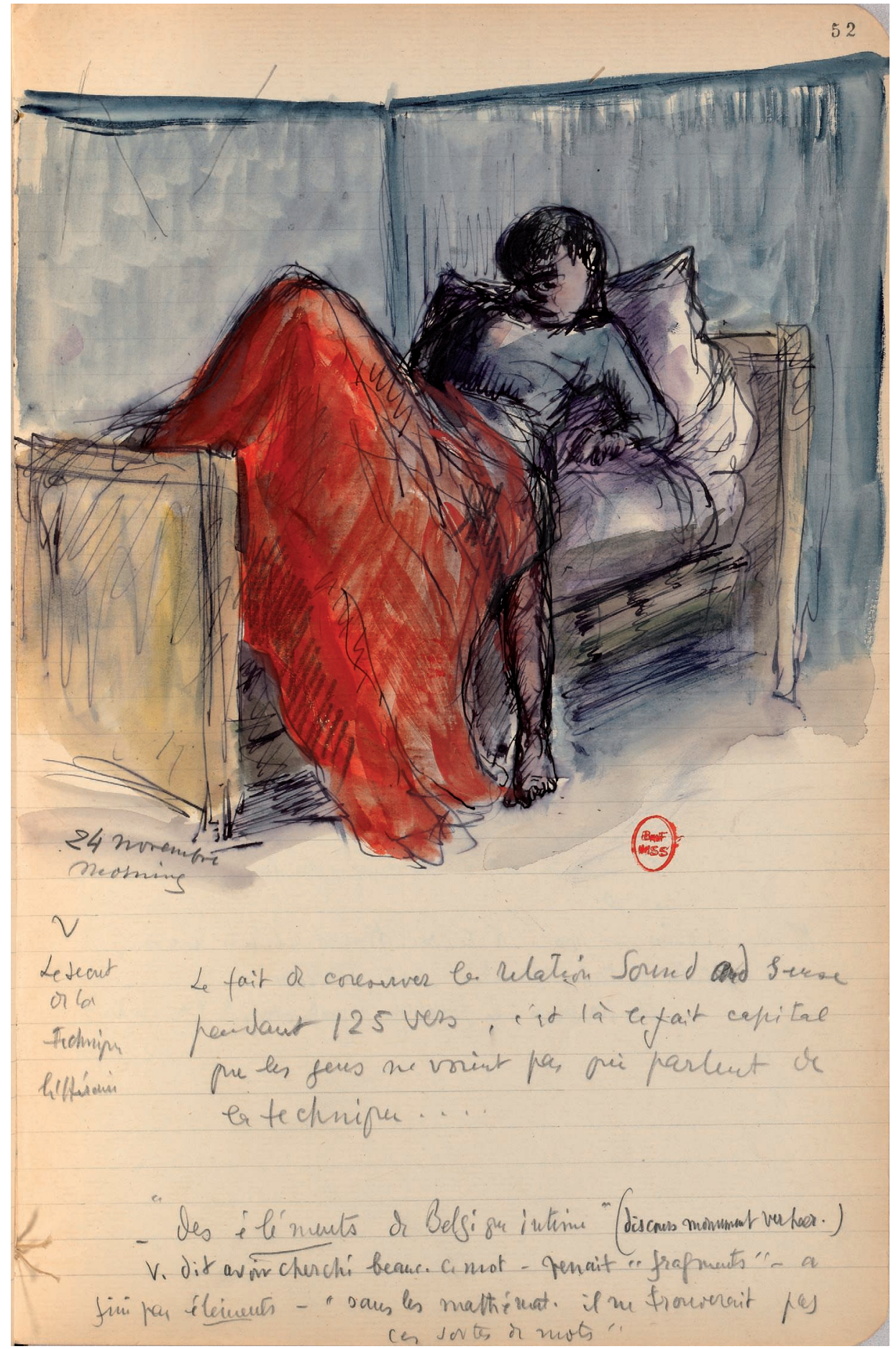

Fig. $10: \mathrm{f}^{\circ} 52 \mathrm{r}^{\circ}$

[marge gauche] 24 novembre morning / V3 Le Secret de la technique littéraire / [corps de texte] Le fait de conserver la relation sound and sense pendant 125 vers, c'est là le fait capital que les gens ne voient pas qui parlent de la technique....

- «des éléments de Belgique intime » (discours monument verhaer ${ }^{4}$.) V. dit avoir cherché beauc. ce mot- venait «fragments » - a fini par éléments - «sans les mathémat. il ne trouverait pas ces sortes de mots »

3. V = Valéry.

4. Verhaeren. Il s'agit du discours prononcé le 10 novembre 1927 à l'occasion de l'inauguration du buste de Verhaeren au square Saint-Séverin. 


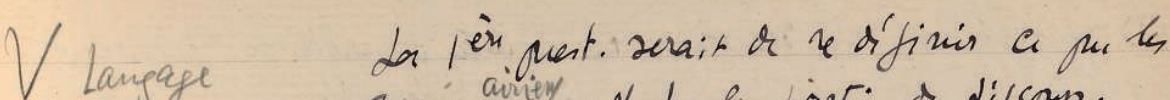
graimin. appellent les partis of discouns.

a madam a madam
Moidann a pelárbajsians
how

la $\mathrm{wm}$

Commols

Sousheristal illusiore su havere Laborat
Ha ldirige ta nageoire

\section{Infusoire, Infusoire,} Viens te poses sur ma main Tu me Jiras le chemin

de la gloire.

bers mon trausparent destin Sois mon dein, mon carlin

mon bignin exfin bénin Sis ma demiès victoire
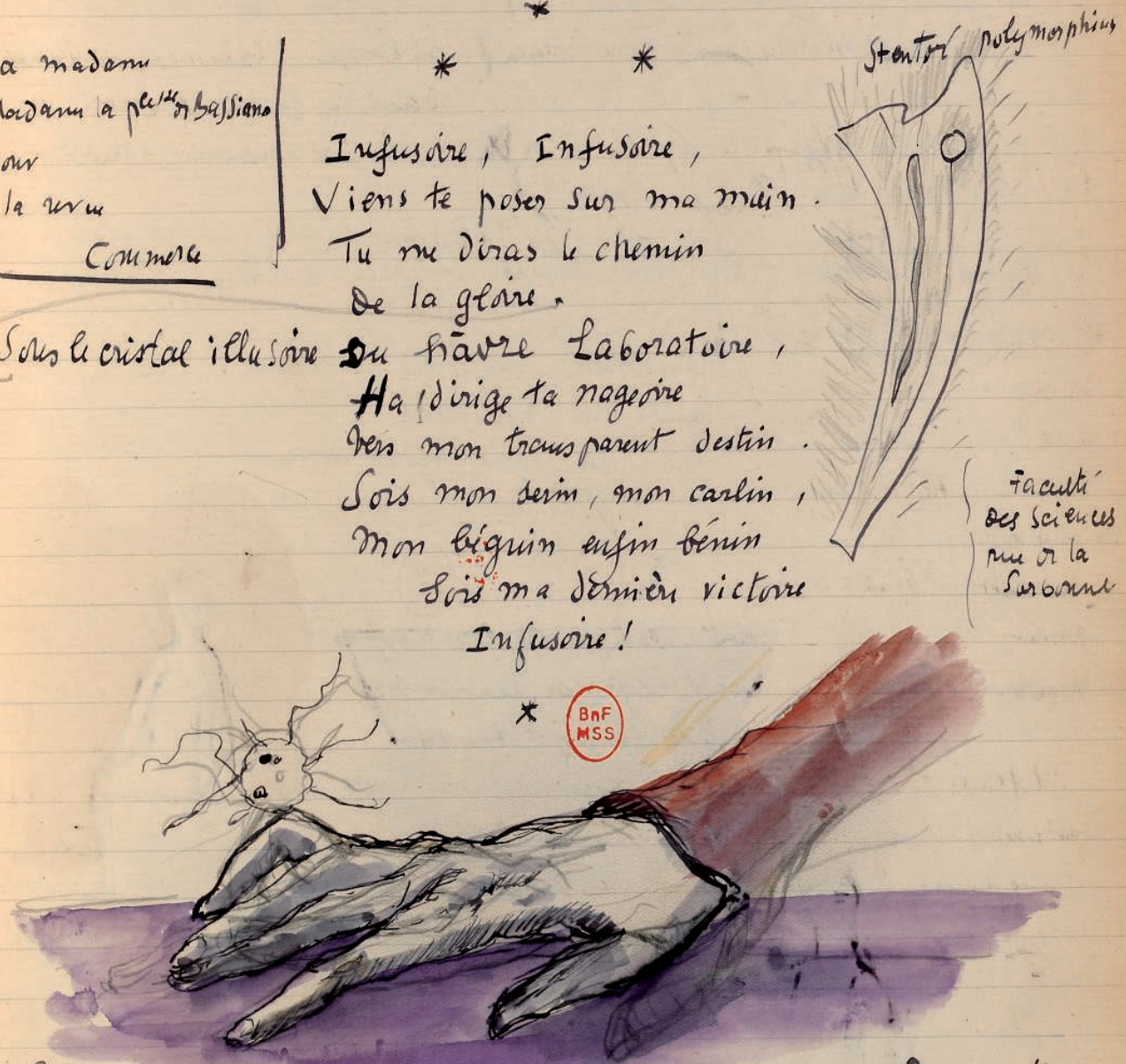

\section{hy riand by Testis}

\section{Geudsiacuder}

vacired

\section{Samodi}
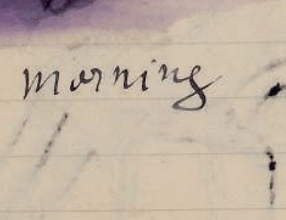

Fig. $11: \mathrm{f}^{\circ} 55 \mathrm{r}^{\circ}$

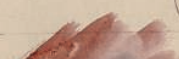

Suroment

V Langage / La 1ère quest. serait de redéfinir ce que les gramm.<airiens> appellent les parties du discours.

[marge gauche] a madame Madame la peesse de Bassiano pour la revue Commerce / [corps de texte] Infusoire, Infusoire,

Viens te poser sur ma main. Tu me diras le chemin de la gloire. <sous le cristal illusoire> du hâvre Laboratoire, Ha, dirige ta nageoire

Vers mon transparent destin. Sois mon serin, mon carlin, Mon béguin enfin bénin Sois ma dernière victoire Infusoire ! / [marge droite] Stentor polymorphin / Faculté des Sciences rue de la Sorbonne

[marge gauche bas] my hand by Testis / [marge droite] Morning 29.11.27

Jeudi académie. Vendredi - conférence $3 \mathrm{~h} / \mathrm{à} 5 \mathrm{~h}$ prix Moréas

Samedi Sorbonne 10 $\mathrm{h}$ matin / déjeuner Bogart et Monod / dîner France-Amérique 


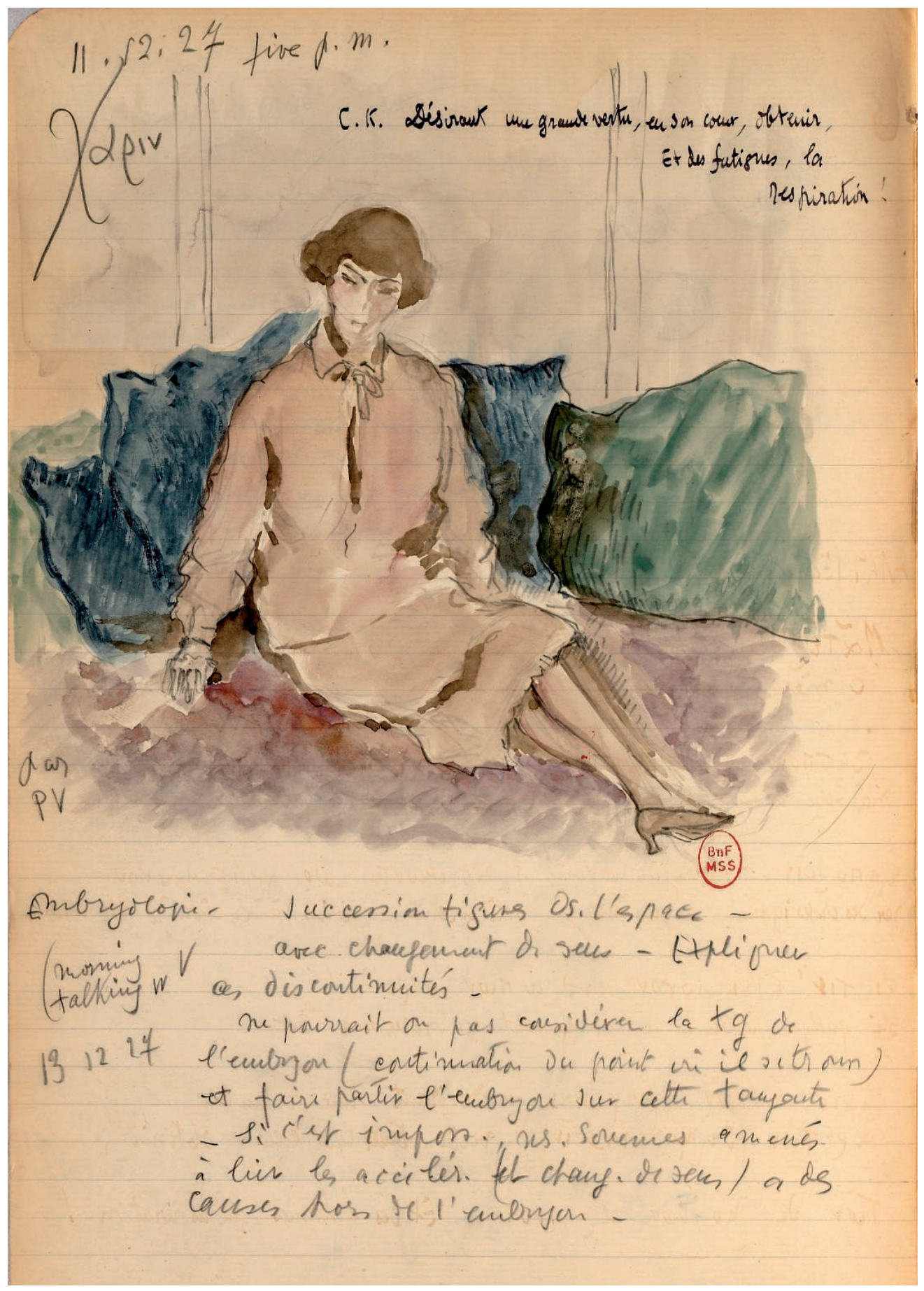

Fig. $12: f^{\circ} 63 v^{\circ}$

11.12.27 five p. $\mathrm{m}$.

Xapiv

C.K. Désirant une grande vertu, en son cœur, obtenir, Et des fatigues, la respiration !

[sous le dessin]

Par PV

Embryologie.

morning

talking w V

Succession figures ds. l'espace -

avec changement de sens - Expliquer ces discontinuités -

ne pourrait on pas considérer la tg de l'embryon (continuation du point où il se trouve)

131227

et faire partir l'embryon sur cette tangente - Si c'est imposs., ns sommes amenés à lier les accélér. (et chang. de sens) à des causes hors de l'embryon. 
Langage

Littérature
déformer le
langage

Les emplois non usuels

du langage -

21.12.27

Science

fait son

langage philosophie

$$
\text { d'un à l'autre }
$$$$
\begin{gathered}
65.5 \\
82.5^{4}
\end{gathered}
$$

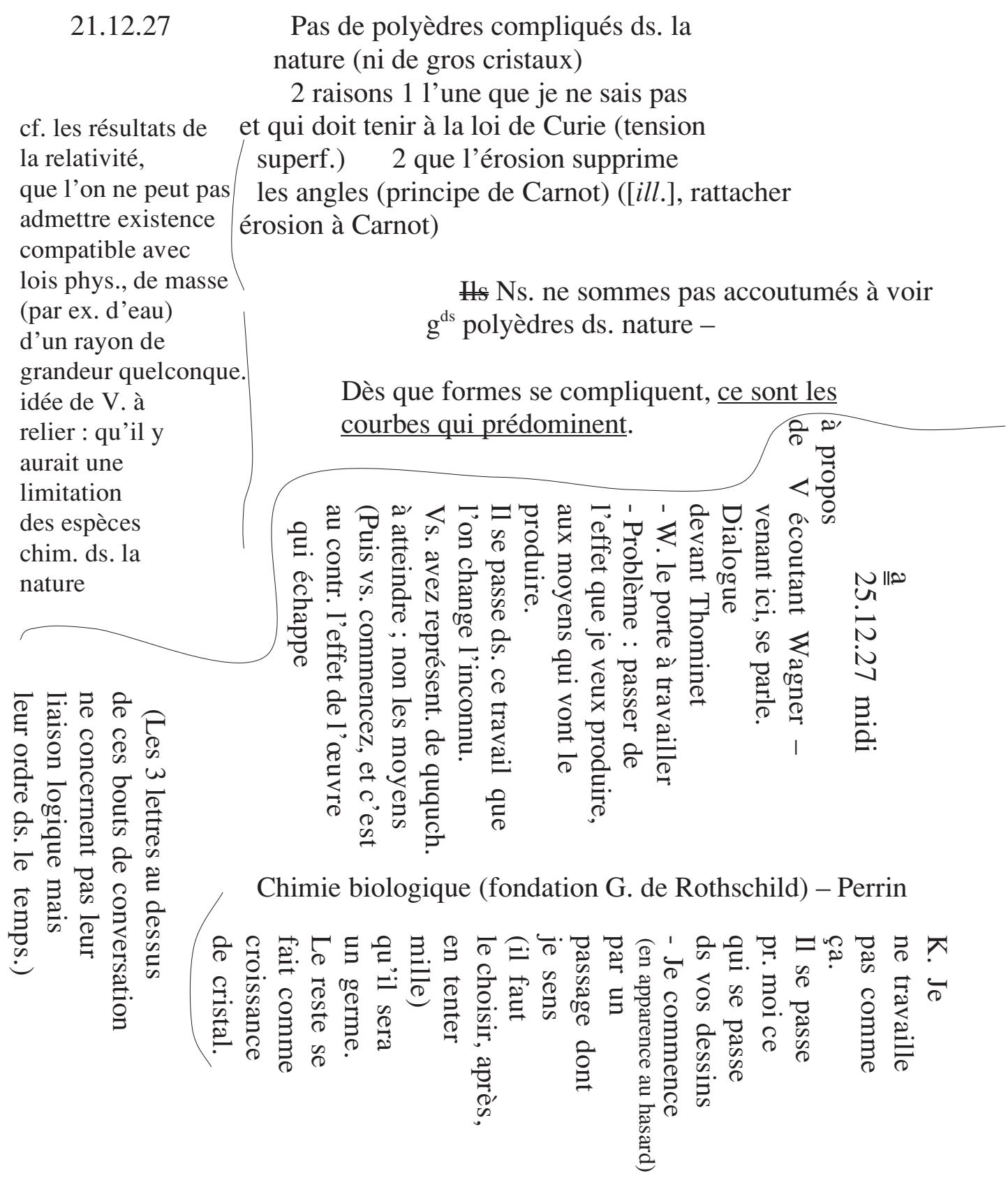




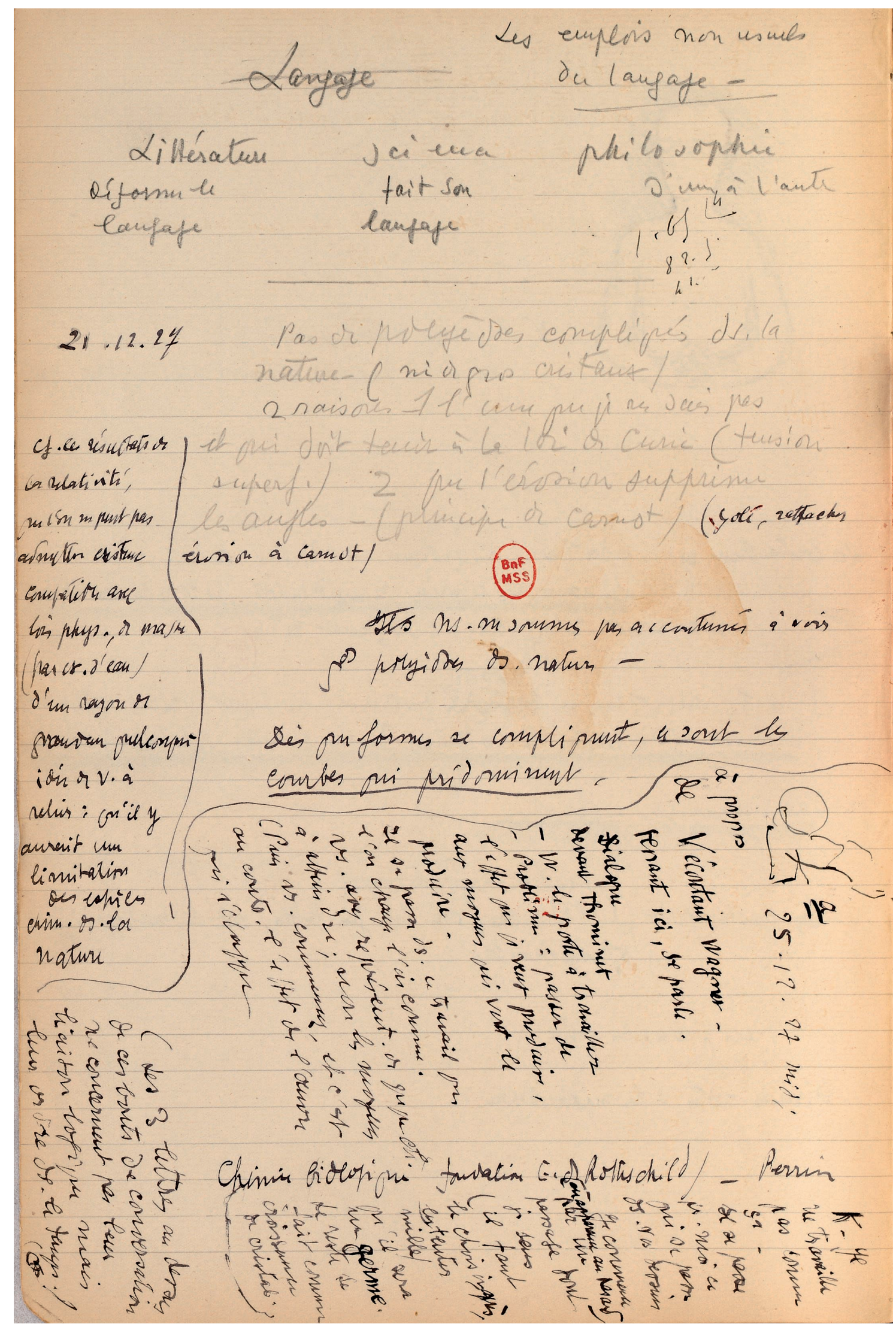

Fig. $13: \mathrm{f}^{\circ} 66 \mathrm{v}^{\circ}$ 


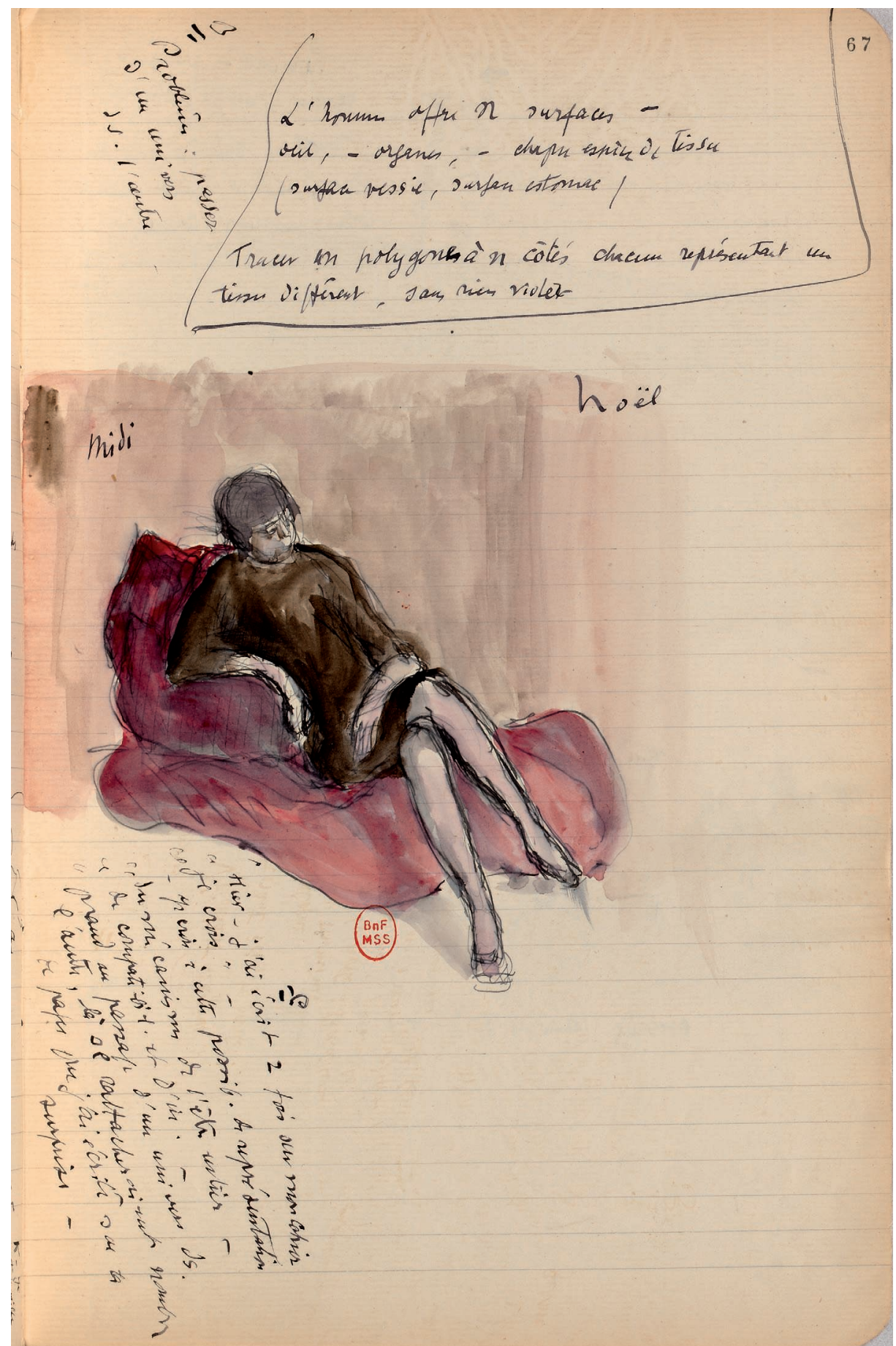

hoül

Fig. $14: f^{\circ} 67 r^{\circ}$

L'homme offre N surfaces - œil, - organes, - chaque espèce de tissu (surface vessie, surface estomac)

Tracer n polygones à $\mathrm{n}$ côtés chacun représentant un tissu différent, sans rien violer

[sur l'aquarelle] midi

Noël

$\underline{B}$

Problème : passer d'un univers ds. l'autre

$\underline{\underline{C}}$

« Hier - j'ai écrit 2 fois sur mon cahier « je crois »-

« Je crois à cette possib. de représentation du mécanisme de l'être entier - de compatibil. et d'in. - quand (sic) au passage d'un univers ds. l'autre, là se rattacheraient nombre de pages que j'ai écrites sur la surprise. 